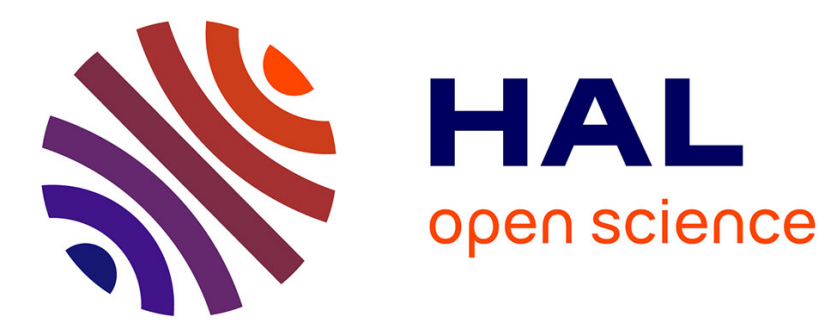

\title{
Speckle-insensitive fringe detection method based on Hilbert transform for self-mixing interferometry
}

\author{
Antonio Luna Arriaga, Francis Bony, Thierry Bosch
}

\section{To cite this version:}

Antonio Luna Arriaga, Francis Bony, Thierry Bosch. Speckle-insensitive fringe detection method based on Hilbert transform for self-mixing interferometry. Applied optics, 2014, 53 (30), pp.69546962. 10.1364/AO.53.006954. hal-01090383

\section{HAL Id: hal-01090383 https://hal.science/hal-01090383}

Submitted on 3 Dec 2014

HAL is a multi-disciplinary open access archive for the deposit and dissemination of scientific research documents, whether they are published or not. The documents may come from teaching and research institutions in France or abroad, or from public or private research centers.
L'archive ouverte pluridisciplinaire HAL, est destinée au dépôt et à la diffusion de documents scientifiques de niveau recherche, publiés ou non, émanant des établissements d'enseignement et de recherche français ou étrangers, des laboratoires publics ou privés. 


\title{
Speckle-insensitive fringe detection method based on Hilbert transform for self-mixing interferometry
}

\author{
Antonio Luna Arriaga, ${ }^{1,2, *}$ Francis Bony, ${ }^{1,2}$ and Thierry Bosch ${ }^{1,2}$ \\ ${ }^{1}$ CNRS, LAAS, 7 avenue du colonel Roche, F-31400 Toulouse, France \\ ${ }^{2}$ Univ de Toulouse, INP, LAAS, F-31400 Toulouse, France
}

compiled: December 3, 2014

\begin{abstract}
A robust phase measurement method to detect self-mixing signals from rough surface targets is addressed. Using the Hilbert transform to create the analytic signal of the monitored optical output power, an accurate instantaneous phase can be extracted without being affected by amplitude variations induced by speckle phenomenon. In case of fringe fading, a running average filter removes the local bias offset improving the calculations. This also allows to observe the phase at dynamic feedback levels within the same calculation, avoiding the need of adding external optical, mechanical or electronic components. This method provides confidence for fringe detection required in subsequent steps to reach sub-wavelength precision of a reconstructed displacement or for velocimetry applications.
\end{abstract}

OCIS codes: $\quad$ (280.3420) Laser sensors; (100.5070) Phase retrieval; (120.3180) Interferometry; (30.6140) Speckle.

http://dx.doi.org/10.1364/XX.99.099999

\section{Introduction}

Self-mixing (SM) interferometry is nowadays an established instrumentation scheme mainly used to measure displacement, absolute distance and velocity of a remote target. The optical output power variations (OOP) obtained from this phenomenon notably related to the amount of backscattered light by the feedback coupling factor $C$, confer a supplemental challenge on the exploitation of these signals as compared to classical interferometers. When a laser diode (LD) is used as source, the poor signal stability caused by the inherent sensitivity of the emitted beam to the back-reflected light has been a limiting factor to spread this technique to perform in-situ accurate measurements. Even minor changes on the requirements specification usually imply major changes and often a complete redesign of this kind of instrumentation scheme [1].

Speckle pattern statistics increase the complexity of exploitation by inducing random amplitude modulation to the already non-linear frequency modulated signal. As the laser spot has finite size, SM signals in this condition exhibit an envelope shape in time domain due to the fading which results of

\footnotetext{
* Corresponding author: alunaarr@laas.fr
}

the incoherent superposition of different waveforms reflected by rough targets. Since each fringe contributes to half LD's wavelength of displacement amplitude, reconstruction of a target movement from SM signals relies on the proper extraction of interferometric features from an acquired signal. If fringes are lost during a measurement, the reconstruction will present proportional inaccuracies regardless the signal processing employed.

To overcome speckle effects, [2] proposed a tracking technique which adjusts the spot on the target by means of piezo-actuators when the received signal amplitude decreases. In a less mechanical context, [3] proposed to use an electronic feedback loop as well as an optical arrangement, allowing to lock the signal measurement to the half of an interferometric fringe. However, the external arrangements used in these solutions dismiss the fully integrated nature of this sensing technique.

With the intention to relax this constraint, [4] introduced the use of an adaptive liquid lens to adjust the beam spot preventing the LD to leave a moderate feedback regime. It is well known in SM literature that this operational mode of SM signals exhibits a characteristic sawtooth-like shape which is easier to exploit algorithmically. However, not all surfaces can provide the necessary amount of 
backscattered light for this working condition, limiting the scope of its applications. A liquid lens was also applied in [5], where signal fading due to speckle was avoided via dynamic focal length variations. Despite the satisfactory impact of this modern component, the reported response time of 80 $\mathrm{ms}$ is not suitable for real-time applications involving large speeds. [5], also proposed to use two LD's with different wavelength and spot size pointing the same target to provide different speckle patterns. Their algorithm alternates signal processing when signal-to-noise ratio decreases in one channel, however this still needs to be further detailed for a realtime implementation.

In a pure algorithmic context, [6] proposed to settle a threshold in adaptive way to increase the scope of an often presented proposal in SM literature based on a phase unwrapping method (PUM from [7]). This convenient notion of having a single method to detect SM signals from a variety of conditions, has been recently improved by [8] to handle noisy conditions by using a predefined piecewise approach. However none of them have been demonstrated convenient to handle random amplitude modulations like the obtained by speckle phenomenon. Let us depict this drawback by referring to Fig. 1 where a simulated SM signal for a moderate feedback regime of $C=2$ and amplitude $6 \lambda_{0}$ has been generated by the behavioral model described in [9], with $\lambda_{0}$ the LD's wavelength emission. The signal has been affected in amplitude with a sinusoidal wave as an attempt to illustrate degradations by speckle pattern found in real measurement environments. In the middle of the signal, it can be observed that those fringes whose amplitude became reduced were not detected by the adaptive threshold algorithm from [6]. This is explained because this approach iterates a threshold over a set of acquired fringes which were assumed to be homogeneous in amplitude.

In order to observe the root issue of this adap-

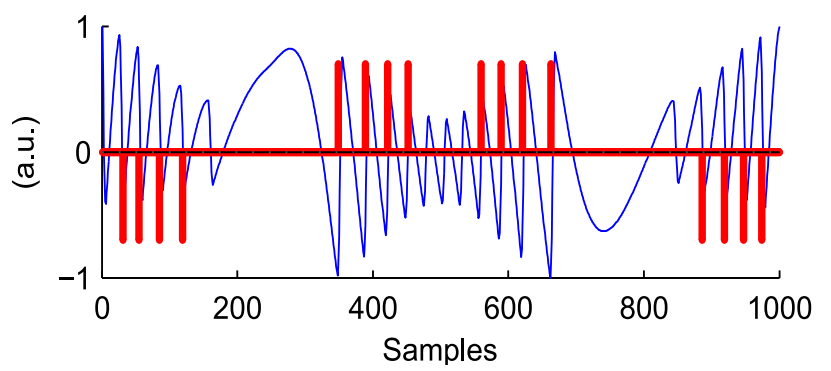

Fig. 1. The algorithm from [6] applied to a simulated SM signal with amplitude modulation. tive threshold proposal, let us outline the common rough phase estimation from [7]. When SM phenomenon occurs, the OOP variations monitored by a built-in photodiode in a LD package (or directly from the junction voltage), are described by:

$$
P(t)=P_{0}\left\{1+m \cos \left[x_{f}(t)\right]\right\}
$$

where $P_{0}$ is the power emitted by the LD in free running state, $m$ is a modulation index, and $x_{f}$ is the interferometric phase subject to back-reflections. The equation linking the original phase $x_{o}$ with $x_{f}$ is given by [11]:

$$
x_{o}(t)=x_{f}(t)+C \sin \left[x_{f}(t)+\arctan (\alpha)\right]
$$

where the parameter $\alpha$ is the LD's linewidth enhancement factor. As demonstrated in [7], by calculating $\alpha$ (usually comprised between 3 and 10) and $C$ (the feedback coupling factor responsible for the varying shape of SM signals), $x_{o}$ can be approached by unwrapping $x_{f}$. A rough estimation of $x_{f}$ can be done by normalizing the OOP from Eq. (1) such as $P_{N}=P(t) / P_{0}$, and applying the inverse cosine function, i.e.:

$$
\hat{x}_{f}(t)=\arccos \left[P_{N}(t)\right]
$$

Once calculated $x_{o}$, the instantaneous distance $D(t)$ between the LD and remote surface which leads to the target's movement can be retrieved from:

$$
x_{o}(t)=\frac{4 \pi D(t)}{\lambda_{o}(t)} .
$$

PUM was originally proposed to work for moderate feedback due to the simplicity of fringe detection and the unambiguity of target's direction retrieval. From Eq. (3) the usage of arccosine function is mathematically correct for rough phase estimation, however it seems to be more pertinent for the case of weak feedback when the fringe's shape is quasi-sinusoidal rather than for sawtooth-like SM signals.

Considering that the work in [10] reported improved precision in the reconstruction process, notably by obtaining an accurate feedback phase with a segmentation procedure, it can be guessed that further improvement on SM signal exploitation might come from a different phase estimation. Also, since identifying random amplitude variations to 
avoid missing fringes is a demanding task, we propose in this work a different approach consisting in the generation of orthogonal SM signals to obtain a robust phase estimation. From this phase signal, it is possible to detect SM fringes in a straightforward manner as this calculation remains homogeneous in amplitude along the time, being thus convenient to perform measurements from rough surface targets.

\section{Robust phase detection}

Recently, [12] presented a detailed analysis on the errors induced by speckle phenomenon over SM signals. From this study, the notion of analytic SM signals obtained through a Hilbert transform has been introduced and enlighten as an intriguing hint to observe speckle phase errors over SM signals.

We have worked with analytic SM signals as a means to estimate a rough phase in a similar manner as the proposal in PUM, i.e. to detect fringe transitions aiming to obtain a practical exploitation of SM signals mainly in the context of displacement measurement.

\section{A. Principle}

We propose to calculate the Hilbert transform $\mathcal{H}$ from the OOP in Eq. (1) in order to produce an orthogonal $90^{\circ}$ phase-shifted SM signal as stated by its theoretical definition [13], i.e.:

$$
P_{a}(t)=P(t)+\operatorname{Im}\{\mathcal{H}[P(t)]\}
$$

where $P_{a}(t)$ is the resulting analytic SM signal.

As a matter of fact, Hilbert transform is used in many fields to provide a frequency information over the time, being suitable for peak detection. In frequency domain it can be calculated by:

$$
\mathcal{F}\{\mathcal{H}[P(t)]\}=j \cdot \operatorname{sgn}(f) \mathcal{F}\{P(t)\}
$$

where $\operatorname{sgn}(f)$, the signum function is defined as 1 for positive frequencies, -1 for negative frequencies and null at zero.

From the analytic SM signal, the instantaneous phase can be calculated as:

$$
\phi(t)=\arctan \left\{\frac{\operatorname{Im}\left[P_{a}(t)\right]}{\operatorname{Re}\left[P_{a}(t)\right]}\right\} .
$$

To illustrate different calculations of analytic SM signals, a sinusoidal displacement of amplitude $4 \lambda_{0}$ has been used to simulate SM signals at increasing feedback levels. Fig. 2(a) corresponds to the calculation of a half period of displacement for weak
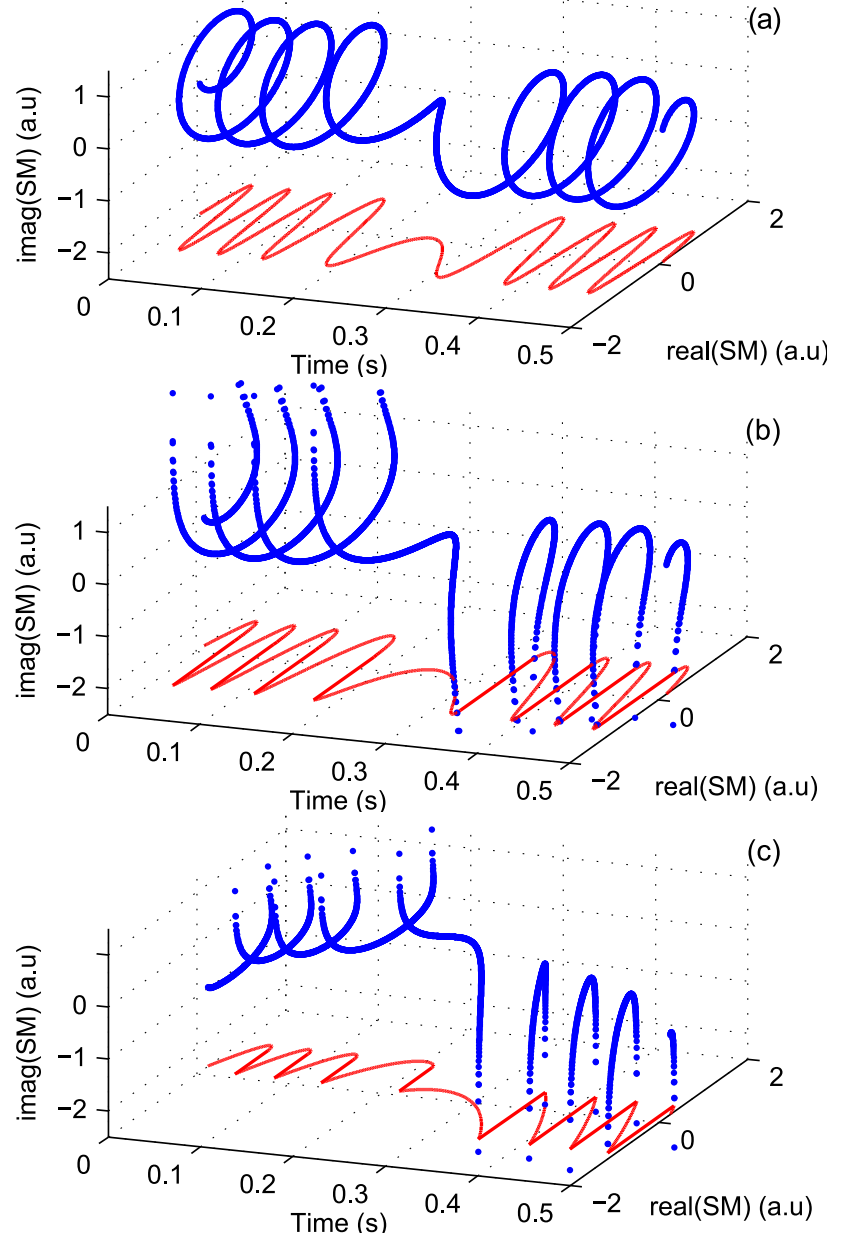

Fig. 2. Analytic SM signals for a sinusoidal displacement of $4 \lambda_{0}$ amplitude, from weak to moderate feedback: (a) $C=0.2$, (b) $C=1.2$ and (c) $C=3.6$.

feedback with $C=0.2$. The bottom projection corresponds to the real axis of SM fringes describing the quasi-sinusoidal shape proper to this regime. Continuing to increase feedback values, in Fig. 2(b) it can be observed that $C=1.2$ (thus in moderate regime), produces a discontinuity on the analytic signal. This allows to confirm the change of regime even if the real SM signal (bottom projection) does not show a so perceptible difference as compared with previous value of $C=0.2$. Finally, a simulation for $C=3.6$ [Fig. 2(c)] reveals a moderate regime by the discontinuity in the waveform, it also reflects hysteresis phenomenon as a more accentuated reduction in amplitude of the observed analytic signal.

From these representations, it can be observed that the calculated analytic form of the SM signal keeps coherent with the fringe's characteristics 
observed in real axis along the different feedback variations. This same behavior can be expected even at different amplitudes of the input SM signal. Therefore, the utility of such an elaboration shall be observed through its phase extraction.

The first observation that can be done from this proposal, is that the acquired SM signal does not need to be normalized as required by the arccosine function from PUM and still produces a similar estimated phase, thus $\hat{x}_{f}(t) \approx \phi(t)$. It can also be seen that the range's phase can be extended to $(-\pi, \pi]$ in order to match the fringe's periodicity of $2 \pi$ by using a four-quadrant arctangent function (arctan2).

The major feature observed from this calculation is the robustness face to amplitude variations as illustrated in Fig. 3(a), where a simulated SM signal for a $10 \lambda_{0}$ displacement and $C=1$ was combined with a sinusoidal signal of unitary amplitude and $0.25 \mathrm{~Hz}$ of frequency. By using the conventional calculation by arccosine function, a mirrored phase mapped version of the input signal was obtained [Fig. 3(b)]. This explains why the adaptive threshold algorithm failed to converge into a low value to properly detect all the SM fringes from the example in Fig. 1. Conversely, Fig. 3(c) demonstrates that the obtained analytic phase (in a range $0-\pi$ to ease the comparative) from Eq. (7) was not affected by amplitude variations.

From the different feedback regimes previously simulated, it can be observed that hysteresis phenomenon produces a deformation on the analytic form of the signals. This implies that the homogeneous phase unwrap of SM fringes is still compromised for the case of fringes from moderate feedback whose zero-crossing has been affected, even if it resulted insensitive to amplitude variations.

We have found that by locally removing the fringe's offset introduced by hysteresis phenomenon, a homogeneous phase calculation can be retrieved like in the case of weak feedback. Fig. 4 presents on the left, a plane view of a small segment with few fringes of the previous analytic calculations for feedback values of $C=0.2,1.2$ and 3.6 from top to down respectively. On the right, from each obtained phase fringe unwrap it can be appreciated how a derivative threshold can be used to detect phase transitions and thus to distinguish the fringes from a signal disregarding their shape in time.

In particular, from the bottom case for $C=3.6$ the zero-mean centered signal on the real axis is contrasted against a hysteresis affected signal to depict the advantage of this procedure. By doing so,
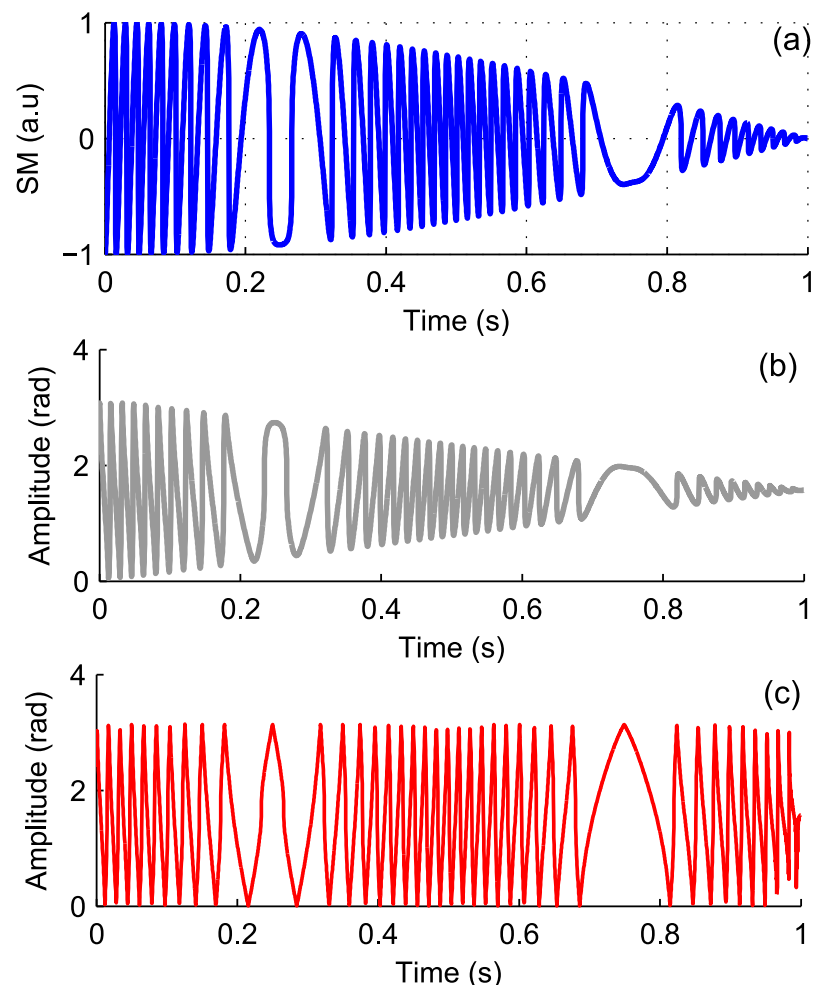

Fig. 3. (a) Simulated SM signal with $C=1$ and amplitude modulation. Comparison between (b) the common phase estimation by arccos, and (c) the proposed instantaneous phase via abs(arctan2).

the iterative procedure of the previously analyzed adaptive threshold algorithm can be avoided.

It can be stated that the combination of hysteresis-free SM signals with their analytic representation through the Hilbert transform provides a means to exploit their phase in a consistent basis even when amplitude variations affect the shape of a group of fringes along the time.

\section{B. Method implementation}

Based on previous analysis, we designed a fringe detection algorithm allowing to increase the scope of Hilbert transform usage in the context of sensors implementation. Since SM signals require a local zero-mean to properly provide an orthogonal calculation, one solution consists in calculating a filter bank for a range of measurements. However, due to the direct dependence of SM fringes to the amount of target's displacement in a period of time, such a filtering requires a self-adapting nature in the context of a generic procedure.

Let us represent in Fig. 5 a comparison between our proposal and the fringe detection basis from PUM algorithm. The analytic signal allows to re- 

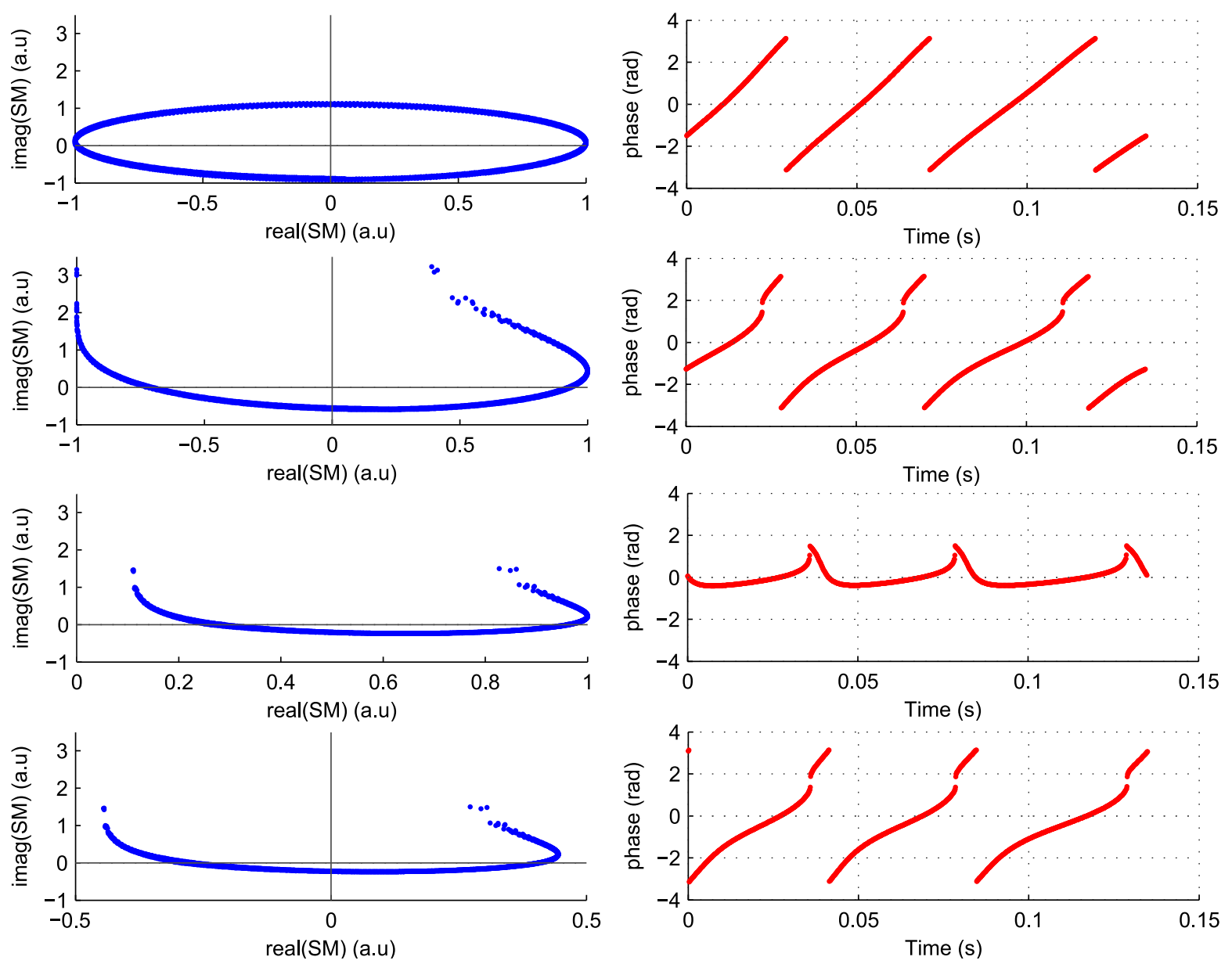

Fig. 4. (on the left) Plane view of few fringes of the analytic SM signals from Fig. 2 and (on the right) the resulting phases for feedback values of $C=0.2,1.2,3.6$ and $C=3.6$ with hysteresis removal, from top to down respectively.

main insensitive to amplitude modulation of the fringes, while the offset removal and direction assignation steps allow to process SM signals regardless their feedback regime.

First, we propose to calculate the derivative from an incoming OOP to obtain a rough observation of SM fringes as usually encountered in SM literature. From this set of points, a root-mean-square (RMS) value can be used to define an automatic threshold aiming to detect the sharpest fringes from a signal. Those detected fringes are then used as trigger points in a running average filter over the original acquired signal. By subtracting this averaged signal to the hysteresis affected signal, it is possible to automatically re-center affected segments $\left(P_{z}\right)$, with the added value of being a noninvasive method for the case of weak feedback as the calculated running average already gives a zero value.

Performing these steps provides thus, a unique algorithm for an improved observation of SM signals' phase. From this calculation it is possible to detect the phase transitions and thus the fringes in a signal. Then, after segmenting the fringes, displacement reconstruction can be achieved by properly assigning the direction to each interferometric fringe $\left(T_{r}\right)$. As a matter of fact, there are several proposals on literature for direction retrieval (e.g. $[7,14])$. A simple calculation consists in assigning the sense directly from the observed differentiated OOP signal and multiplying this value by the detected phase transition instants from previous step $\left(\Delta \hat{x}_{f}\right)$.

In order to validate the contribution of our generic fringe detection algorithm, it has been compared against the unfavorable conditions tested in Fig. 1 for the adaptive threshold algorithm from [6]. In Fig. 6, the SM signal used to observe the issue of undetected fringes due to amplitude variations has been used as input to our algorithm $(C=2$, displacement of $6 \lambda_{0}$ and with fringes modulated in amplitude). For convenience, it has been denoted HT since it relies on a Hilbert transform calculation 


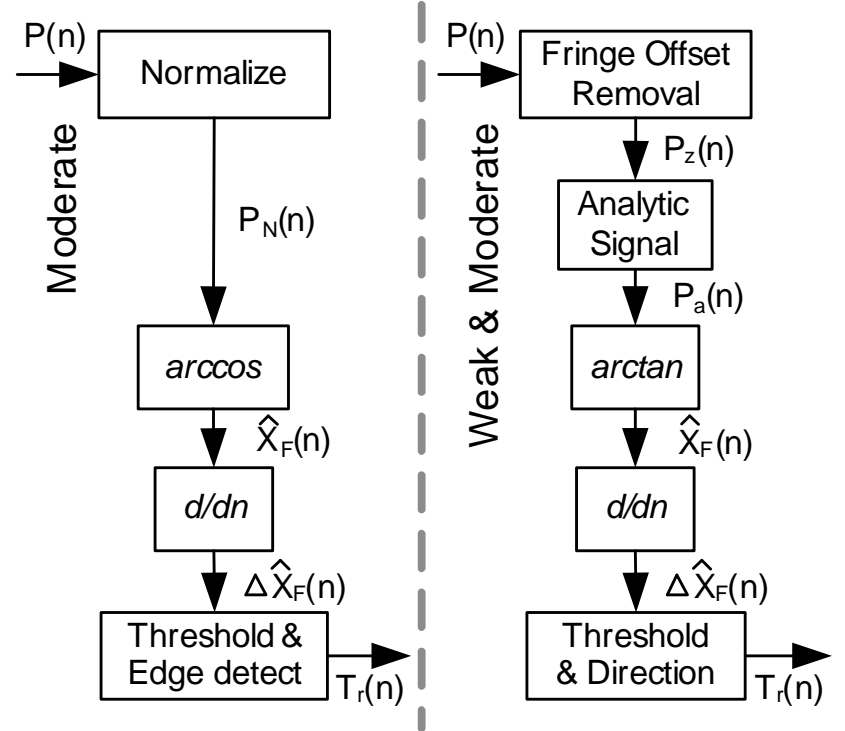

Fig. 5. Fringe detection basis from PUM algorithm [7] compared to our proposal based on Hilbert transform.

to obtain the analytic form of the SM signal (which is then used for improved phase calculation).

As depicted in Fig. 6(a), our proposal has been able to successfully account all the fringes in the signal by exploiting its improved phase calculation scheme. Fig. 6(b) compares the impact of failing to detect all the fringes from a SM signal in the case of a simple fringe counting procedure. For this example, the 3 missed fringes from the adaptive threshold algorithm carry an error of $1.5 \lambda_{0}$ peak-to-peak. As depicted in Fig. 6(c), those undetected fringes directly affect a displacement reconstruction procedure like the case of PUM minimization algorithm which is able to reach precisions below $20 \mathrm{~nm}$ when all the fringes are properly detected.

Other than the feature of working with different fringe amplitudes, it is important to highlight from this use case that the iterative feature and normalization step from the adaptive threshold algorithm have been conveniently simplified by the analytic SM signal elaboration. Since Hilbert transform can be implemented as a filtering procedure, this proposed approach is convenient for real-time calculations by conventional signal processing hardware architectures, similarly to the implementation of fast Fourier transforms.

\section{Experiments and discussion}

The experimental setup is shown in Fig. 7. A Hitachi HL7851G LD, with wavelength $\lambda_{0}=785 \mathrm{~nm}$ is powered in continuous wave at $20 \mathrm{~mW}$. The emitted beam is collimated through a lens with focal length of $5 \mathrm{~mm}$ and focalized to a random point on
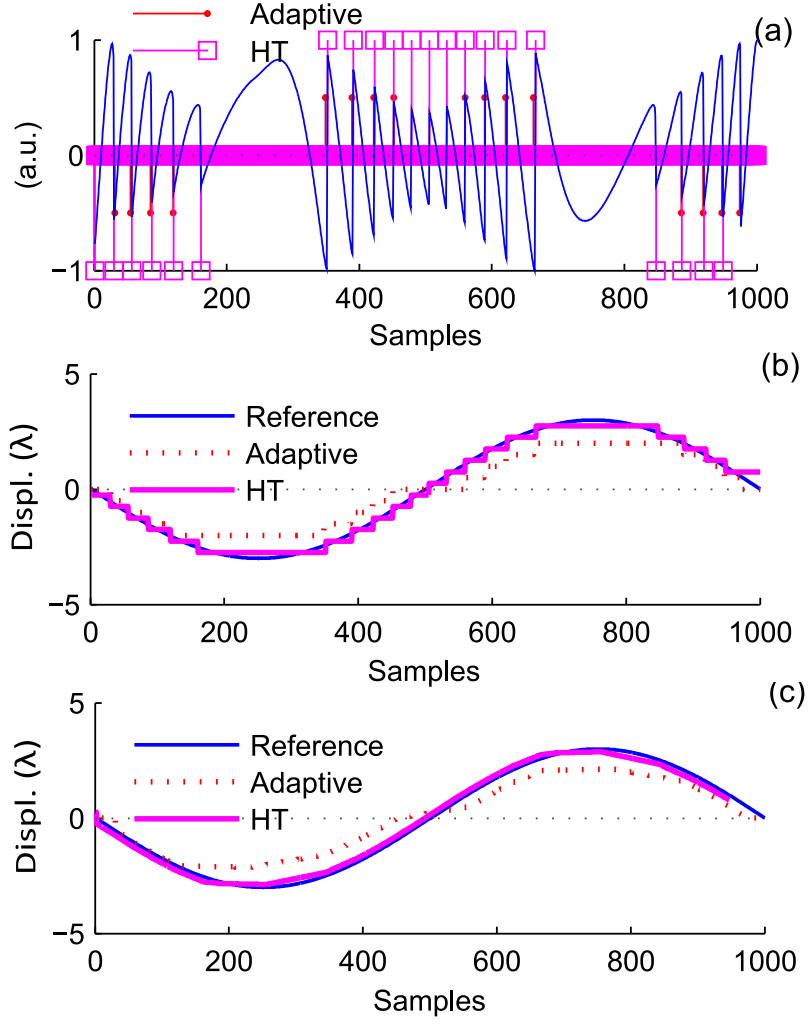

Fig. 6. Comparative between the adaptive threshold algorithm and our proposal: (a) Detected fringes, (b) Unwrapped fringe counting, and (c) Displacement reconstruction.

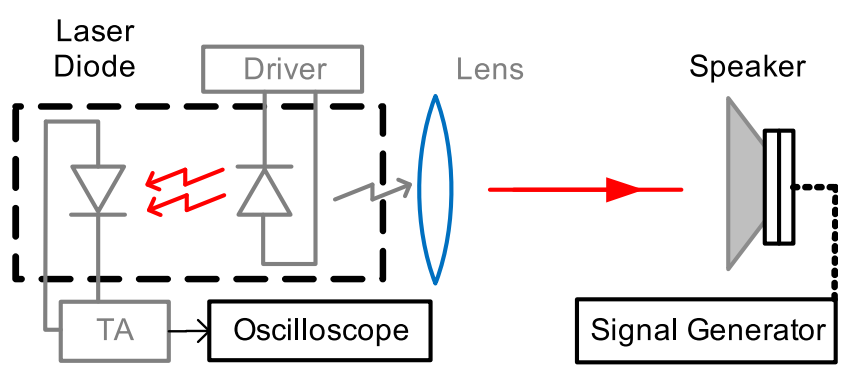

Fig. 7. Experimental setup for displacement measurement by SM and speckle patterns emergence.

a speaker with diameter of $80 \mathrm{~mm}$. The distance from the LD to target is $45 \mathrm{~cm}$.

The speaker is excited by a sinusoidal wave of 300 $\mathrm{Hz}$ and $10 \mathrm{Vpp}$ from a Tektronix AFG3022 function generator. In a first experience, a surface area is coated with sandpaper P40 of $425 \mu \mathrm{m}$ average roughness. In a second experience, the coating is a slow-fading phosphor material active in the IR (VRC2 from Thorlabs). This intentional coarse arrangement leads to speckle emergence over micrometric displacement as it would be on a real nonconditioned environment. The HL7851G embeds a photodiode which serves to monitor the LD's OOP 
variations. By means of a transimpedance amplifier (TA) and an oscilloscope (LeCroy HRO 66Zi) we observe and record the SM signals to apply our fringe detection algorithm and perform a displacement reconstruction as described hereafter.

\section{A. Speckle affected SM displacement signal}

Figure 8, shows the acquired SM signal for one period of the sandpaper-coated surface moving towards and away the LD. These fringes present in average a sawtooth-like shape, however it can be appreciated an abrupt amplitude degradation due to speckle phenomenon. As explained, this unpredictable variation is a main source of error in a measurement by this technique. For example, it can be observed in Fig. 9(a-b) that settling a threshold over a set of derived fringes is just an approach to partially extract some information from a SM signal, however it is not convenient as final solution due to the fringes that are not detected when the amplitude changes over a few of them. This is why we have considered this information just as a preliminary step, notice that the region of undetected pseudo-fringes actually shares a similar average value around zero for this experimental signal.

Performing the moving average over such detected pseudo-fringes in this signal allows to observe the non-intrusive nature of this approach, as the fringes were already zero-centered. i.e. the acquired SM signal was in moderate regime without hysteresis phenomenon [Fig. 9(c)]. In this condition, the analytic SM form was created by using the Hilbert transform allowing the improved phase observation instead of the arccosine function which would provide a mirrored amplitude modulated signal. From the homogeneous calculated phase, its derivative provided the transitions denoting each of the fringes. Their direction was assigned by multiplying them with the sign of the fluctuations of the original SM derivative [Fig. 9(d)]. Finally, target displacement reconstruction at $300 \mathrm{~Hz}$ has been

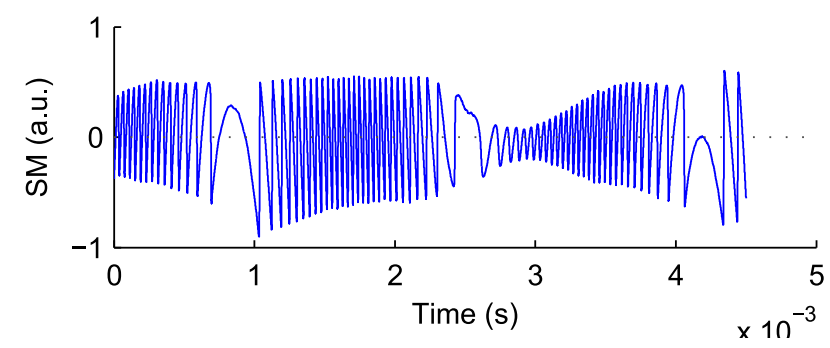

Fig. 8. One period of a speckle affected signal for displacement retrieval.

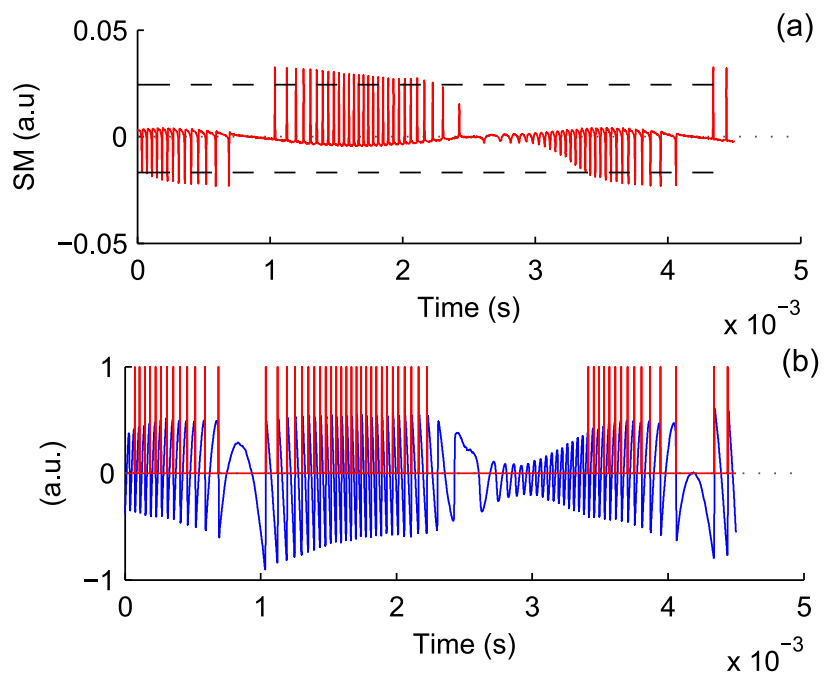

(a)
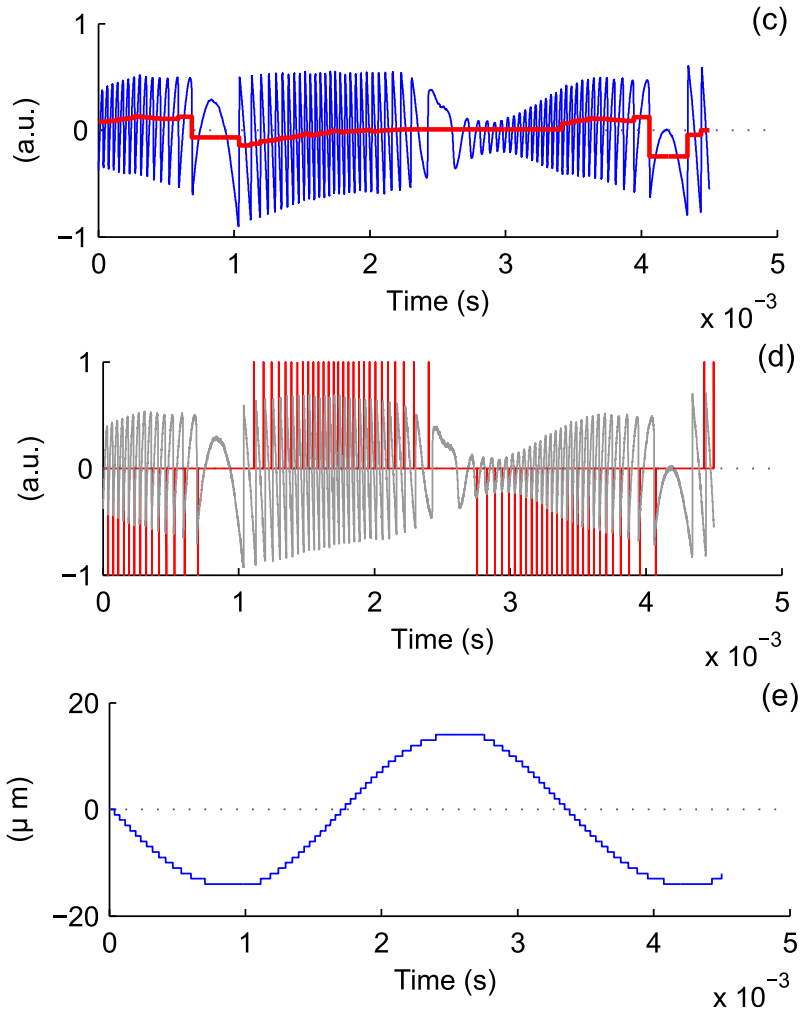

Fig. 9. Steps of the proposed method for fringe detection for SM displacement reconstruction: (a) Derivative, (b) Pseudo-fringes, (c) Offset removal, (d) Phase transitions, and (e) Displacement reconstruction.

achieved properly as observed in Fig. 9(e). Let us highlight that the goal has been to validate the pertinence of the fringe detection scheme over the existing literature. In near future it is expected to propose another test with a countermeasure for a comparative validation. For convenience, let us summarize the performed steps over the acquired signal as follows: 
1. Derive the OOP signal and calculate its RMS [Fig. 9(a)].

2. Define as trigger points those values above the RMS [Fig. 9(b)].

3. Perform signal average between each previous points and subtract it from OOP [Fig. 9(c)].

4. Create the analytic signal from Eq. (5) by using a Hilbert transform.

5. Calculate the phase in $(-\pi, \pi]$ range from Eq. (7) as shown in Fig. 4.

6. Select the phase transitions in a $\bmod [2 \pi]$ basis and assign a positive or negative value according to target direction [Fig. 9(d)].

7. Reconstruct target's displacement from those assigned transitions [Fig. 9(e)].

As previously explained, for this last point a precise displacement reconstruction can be done for example by the optimization algorithm from PUM, once that all the fringes have been properly detected.

\section{B. Worst usage conditions}

In the second experience, pointing the phosphorcoated area produced stronger amplitude degradations leading to a fringe fading [Fig. 10(a)].

Direction assignation by the simple differentiation approach provided a small segment of detected fringes in opposite sense to the rest of surrounding fringes within half period of target's displacement [Fig. 10(b)]. In fact, looking closely to the segments of fringe fading [Fig. 10(c)], it can be observed a weak feedback regime from the quasi-sinusoidal shape while the signal in general depicts sharpened sawtooth fringes. While this condition of dynamic feedback variation leads to a very complex identification, our approach which disregards the shape in time of fringes allows to observe their phase, even if direction assignation of a few of them still requires further improvement. It can also be observed that the single fringe of smallest amplitude (located at $\approx 3.2 \mathrm{~ms}$ ) was not detected as it was almost equivalent to the signal noise amplitude. As mentioned in the introduction, the implication in both cases is an inaccuracy of the reconstruction. However those wrong detections can be corrected by improving this assignation step.

In Fig. 11(a), a statistical direction was assigned to those detected fringes by analyzing its average separation and accounting the surrounding detections in similar way as a visual inspection producing a smooth displacement reconstruction [Fig.
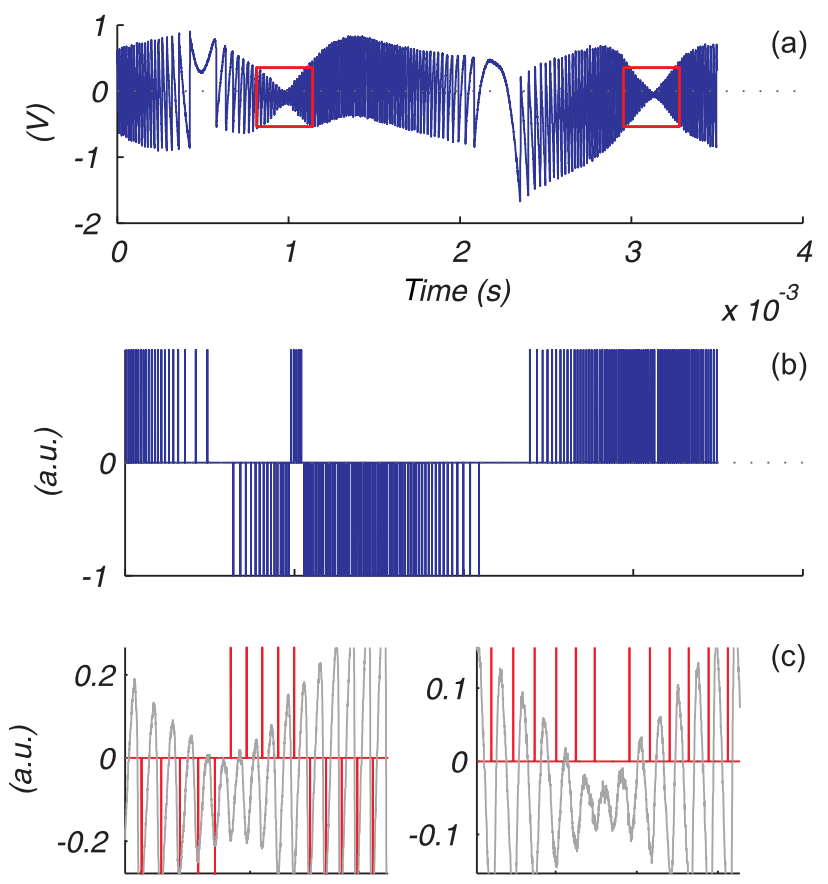

(c)

Fig. 10. Strong speckle condition: (a) Input signal, (b) Detected fringes, and (c) zoom on input signal depicting dynamic variations of feedback regime.

11(b)]. As can be observed in a contemporary application for target speed with direction measurement from SM signals in frequency domain [15], direction retrieval of single fringes degraded by noise and speckle still requires further signal processing research. While this ambiguity could be tolerated for several applications (e.g. displacements above the tens of $\mu \mathrm{m}$ ), an increased processing complexity shall be expected to attain improved performance.

\section{C. Application to velocimetry}

In order to observe the viability of our proposal for real usage in extended applications, a test for fringe detection has been proposed with a basic velocimetry setup, where a distributed feedback (DFB) laser diode points the rough surface of a disk rotating at constant velocity in one direction [Fig. 12(a)].

An experimental signal segment [Fig. 12(b)] was acquired to observe the speckle phenomenon as a random amplitude modulation strongly affecting SM fringes. For clarity, just a part of this signal [Fig. 12(c)] has been passed to our algorithm and to the adaptive threshold algorithm for comparison. Looking closely to the fringes, it can be seen that those with bigger amplitude present a characteristic sawtooth-like shape allowing a simple target 


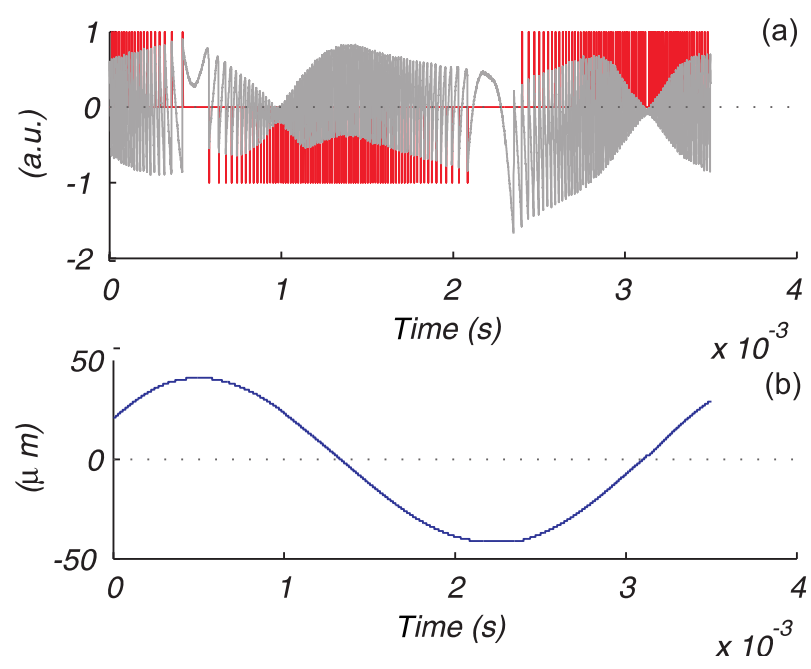

Fig. 11. (a) Improved detection of fringes under strong speckle, and (b) Displacement reconstruction from them.

direction identification. On the other hand, those with smaller amplitude become more triangular and is almost impossible to observe a privileged sense (even if it is known that the target rotates only in one direction).

The obtained transitions from both compared algorithms are shown in Fig. 12(d). Those corresponding to the adaptive algorithm (scaled down for clearness) present few gaps for undetected values after a threshold settlement. Conversely, after denoising the input segment our algorithm detected all the fringes demonstrating thus its robustness.

\section{Conclusion}

The presented phase calculation approach allows to detect SM fringes regardless the dynamic variations in the feedback level even face to speckle degradations. The robustness of this detection scheme resides in the fact that it allies the benefits of frequency domain signal exploitation through the Hilbert transform, with the flexibility of dealing with SM fringes in time domain.

This method has allowed to derive a convenient algorithm for displacement reconstruction, avoiding the need of external arrangements and maintaining low the global cost of system development based on SM interferometry. Due to its versatility and capabilities, it shall be studied for displacements in the range of $\mu \mathrm{m}$ to $\mathrm{mm}$, in unconditioned environments, and can be applied to related applications of SM interferometry like velocimetry.

The corresponding author would like to thank Clement Tronche for experimental setups and CONACYT for financial support.
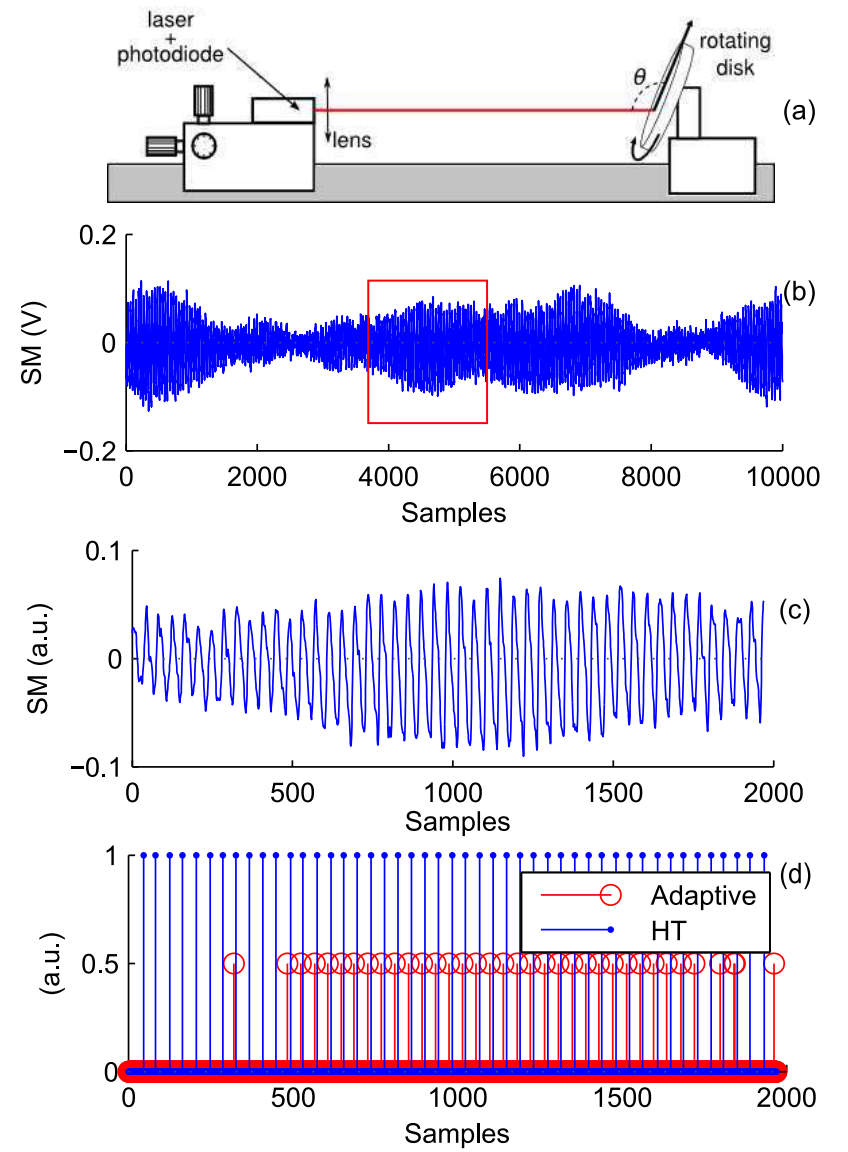

Fig. 12. (a) Experimental velocimetry setup with a rotating disk, (b) Speckle affected fringes at constant frequency, (c) Algorithms' input signal for validation, and (d) Comparison of detected transitions.

\section{References}

[1] S. Donati, "Developing self-mixing interferometry for instrumentation and measurements," Laser Photonics 6, $393-417$ (2012).

[2] M. Norgia, S. Donati, and D. D'Alessandro, "Interferometric measurements of displacement on a diffusing target by a speckle tracking technique," IEEE Journal of Quantum Electronics 37, 800-8006 (2001).

[3] G. Giuliani, S. Bozzi-Pietra, and S. Donati, "Selfmixing laser diode vibrometer," Measurement Science and Technology 14, $24-32$ (2003).

[4] U. Zabit, R. Atashkhooei, T. Bosch, S. Royo, F. Bony, and A. D. Rakic, "Adaptive self-mixing vibrometer based on a liquid lens," Optics Letters 14, $1278-1280$ (2010).

[5] R. Atashkhooei, S. Royo, and F. Azcona, "Dealing with speckle effects in self-mixing interferometry measurements," IEEE Sensors Journal 13, 16411647 (2013).

[6] U. Zabit, T. Bosch, and F. Bony, "Adaptive transi- 
tion detection algorithm for a self-mixing displacement sensor," IEEE Sensors Journal 9, 1879 - 1886 (2009).

[7] C. Bes, G. Plantier, and T. Bosch, "Displacement measurements using a self-mixing laser diode under moderate feedback," IEEE Transactions on Instrumentation and Measurement 55, 1101-1105 (2006).

[8] Z. Huang, C. Li, and X. Sun, "Piece-wise transition detection algorithm for a self-mixing displacement sensor," Chinese Optics Letters 11, 091203 (2013).

[9] G. Plantier, C. Bes, and T. Bosch, "Behavioral model of a self-mixing laser diode sensor," IEEE Journal of Quantum Electronics 41, 1157-1167 (2005).

[10] Y. Fan, Y. Yu, J. Xi, and J. F. Chicharo, "Improving the measurement performance for a self-mixing interferometry-based displacement sensing system," Applied Optics 50, 5064-5072 (2011).
[11] D. M. Cane and K. A. Shore, eds., Unlocking Dynamical Diversity: Optical Feedback Effects on Semiconductors Lasers (Wiley, 2005).

[12] S. Donati, G. Martini, and T. Tambosso, "Speckle pattern errors in self-mixing interferometry," IEEE Journal of Quantum Electronics 49, 798-806 (2013).

[13] R. N. Bracewell, The Fourier transform and its applications (McGraw-Hill, 1978).

[14] A. Magnani, A. Pesatori, and M. Norgia, "Selfmixing vibrometer with real-time digital signal elaboration," Applied Optics 51, 5318 - 5325 (2012).

[15] A. Magnani, A. Pesatori, and M. Norgia, "Real-time self-mixing interferometer for long distances," IEEE Transactions on Instrumentation and Measurement 63, 1804-1809 (2014). 